\title{
Accretion shock signatures in the spectrum of two-temperature advective flows around black holes
}

\author{
S. Mandal ${ }^{1}$ and S. K. Chakrabarti ${ }^{2,1}$ \\ ${ }^{1}$ Centre for Space Physics, Chalantika 43, Garia Station Rd., Garia, Kolkata 700084, India \\ e-mail: space_phys@vsnl.com \\ 2 S.N. Bose National Centre for Basic Sciences, JD Block, Salt Lake, Sector III, Kolkata 700098, India \\ e-mail: chakraba@bose.res.in
}

Received 6 May 2004 / Accepted 30 December 2004

\begin{abstract}
The centrifugal barrier supported boundary layer (CENBOL) of a black hole affects the spectrum exactly in the same way the boundary layer of a neutron star does. The CENBOL is caused by standing or oscillating shock waves that accelerate electrons very efficiently and produce a power-law distribution. The accelerated particles in turn emit synchrotron radiation in the presence of the magnetic field. We study the spectral properties of an accretion disk as a function of shock strength, compression ratio, flow accretion rate and flow geometry. In the absence of a satisfactory description of magnetic fields inside the advective disk, we only consider the stochastic fields and use the ratio of field energy density to gravitational energy density as a parameter. Not surprisingly, stronger fields produce larger humps due to synchrotron radiation. We not only include "conventional" synchrotron emission and Comptonization due to Maxwell-Boltzmann electrons in the gas, but also compute the effects of power-law electrons. For strong shocks, a bump is produced just above the synchrotron self-absorption frequency at $v_{\text {bump }} \sim v_{\text {inj }}\left[1+\frac{4}{3} \frac{R-1}{R} \frac{1}{x_{\mathrm{s}}^{1 / 2}}\right]_{\mathrm{s}}^{1 / 2}$, where, $v_{\text {inj }}$ is the frequency of the dominant photons from the pre-shock flow, and $R$ the compression ratio of the shock located at $x_{\mathrm{s}}$. For strong shocks, a bump at a higher frequency appears predominantly due to the power-law electrons formed at the shock front.
\end{abstract}

Key words. black hole physics - accretion, accretion disks - hydrodynamics - radiative transfer - shock waves

\section{Introduction}

Attempts to explain the spectra of black holes were made in the early seventies (Shapiro 1973a,b) by using spherically symmetric Bondi flows (Bondi 1952), but the flow was found to be very radiatively inefficient due to high advection. In the same year Shakura-Sunyaev (1973) proposed a disk model in which the angular momentum distribution is kept as the Keplerian distribution around a black hole. However, emitted multi-colour black body radiation was unable to explain the spectra of Cyg X-1 (Eardley et al. 1975). Shapiro et al. (1976) went on to show that it is possible to have a "hotter" branch of the solution and still retain Keplerian distribution, which can produce hard X-rays from the inner region. On the other hand, Eardley \& Lightman (1975) found that a Keplerian disk with constant viscosity parameter $\alpha_{\text {vis }}$ was unstable due to thermal and viscous effects (1975), which was later corroborated by Eggum et al. (1985) in their detailed numerical simulation. They showed that the Keplerian disk collapsed in the presence of a constant $\alpha_{\text {vis. }}$. The focus was then to introduce a mixture of sub-Keplerian and super-Keplerian (non-accreting) flows, such as thick accretion disk in the optically thick limit (Paczyński \& Wiita 1980) or accretion torus in the optically thin limit (Rees et al. 1982), and their spectral properties were studied (e.g.,
Madau 1988). Other models included "non-Keplerian" components, such as magnetic corona (Galeev et al. 1979). At the same time, the sub-Keplerian transonic flow models were introduced (Muchotrzeb \& Paczyński 1982) and improved upon to include standing, oscillating or propagating shock waves (e.g. Chakrabarti 1989; Chakrabarti \& Molteni 1995; Molteni et al. 1996). Subsequently, Chakrabarti \& Titarchuk (1995, hereafter CT95) incorporated this flow solution in computing spectra of black holes. In recent years, it has become customary to include the sub-Keplerian flows in accretion flow models because of compelling evidence that the Keplerian disk alone cannot explain most of the observational facts and that two components were essential (Smith et al. 2002, 2001; see Chakrabarti 1996, for a review). There are several other models in the literature which also include a secondary component such as a Compton cloud or magnetic corona (Poutanen \& Coppi 1998; Gierliński et al. 1999; Coppi 1999; Zdziarski et al. 2001), which satisfactorily fit spectra up to high energies as well. Esin et al. (1998) fits spectra with an ADAF model in which the Keplerian flow becomes ion pressure dominated in the inner region. In the present paper, we shall concentrate on spectral properties of the dynamic "Compton cloud", namely, the sub-Keplerian transonic flow component, and not deal with other models already present in the literature. 
CT95 introduces a two component advective flow (TCAF) solution in which the Keplerian disk was on the equatorial plane, as usual, but a dynamic sub-Keplerian halo component (basically the transonic solutions worked out earlier, see Chakrabarti 1996) flanks this Keplerian disk. While the Keplerian disk was supplying soft-photons, the sub-Keplerian flow was supplying hot electrons; and the relative importance of these two accretion rates determined whether the spectrum was going to be soft or hard. A pivotal role is played in this context by the CENtrifugal barrier supported BOundary Layer, or CENBOL. This is the hot and puffed-up, post-shock region which intercepts soft-photons from the Keplerian disk and reprocesses them through inverse Comptonization. It is to be remembered that the CENBOL is a direct consequence of the dominant centrifugal force near a black hole that is not to be confused with either the puffed up corona or magnetized corona used by other models. We find it comfortable to use CENBOL as the Comptonizing cloud since its existence and stability have been shown beyond doubt in a large number of numerical simulations (Molteni et al. 1994; Chakrabarti \& Molteni 1995; Molteni et al. 1996), and since non-axisymmetric perturbations also could not remove them, (see Molteni et al. 1999). We can produce the observed power-law component without resorting to any hypothetical electron cloud. In essence, the CENBOL region in between the horizon and the shock behaves like a boundary layer where the flow dissipates its gravitational energy. This boundary layer can oscillate when cooling is introduced, so this is proposed (Molteni et al. 1996; Chakrabarti \& Manickam 2000; Chakrabarti et al. 2004) as the cause of the Quasi-Periodic Oscillation.

Given that a significant fraction of the accretion flow could pass through a standing, oscillating or a propagating shock, it is likely that the hot electrons may be accelerated by them, just as high energy cosmic rays (see Kazanas \& Elision 1986; Molteni \& Sponholz 1994; Chakrabarti 1996) are produced by transient super-novae shocks (Bell 1978a,b; Longair 1981). Our analysis incorporates the power-law component of the electrons to compute the synchrotron radiation and the Comptonization of photons by these electrons. Inclusion of the synchrotron emission generated by non-thermal electrons is not new. For example, Lin \& Liang (1999) consider a hybrid model which includes a few percent of non-thermal electrons, along with the usual thermal electrons obeying Maxwell-Boltzmann distribution. The emission included thermal and magnetic bremsstrahlung but no Comptonization. In a parametric study, with the percentage of non-thermal electrons as a free parameter, Wardzinski \& Zdziarski (2001) show that the soft photons generated could significantly change the spectra. These works do not address the origin of the non-thermal electrons quantitatively but propose that they could be produced in magnetized corona. In contrast, we assume that all the non-thermal electrons are produced in the accretion shocks, although some electrons from magnetized corona could not be ruled out and should be included for a completely consistent solution. Some examples of changes in the spectral states of the black holes are presented in Mandal \& Chakrabarti (2004).

In the next section, we present the basic equations and discuss the relevant parameters. We first assume the flow to be around a Schwarzschild black hole. For the sake of simplicity, we use the well-known Paczyński \& Wiita (1980) pseudoNewtonian potential $\left(\phi_{\mathrm{PN}}=-\frac{G M}{r-r_{\mathrm{g}}}\right.$, where $r_{\mathrm{g}}=2 G M / c^{2}$ is the Schwarzschild radius of the black hole, $M$ the mass of the black hole, $G$ and $c$ are the universal gravitational constant and the velocity of light, respectively) to describe the black hole geometry. In Sect. 3, we discuss the nature of heating and cooling processes in an advective flow. In Sect. 4, we present our procedure to compute the Comptonized spectra using powerlaw electrons. In Sect. 5, we explain the solution procedure, while in Sect. 6, we present the emergent spectra for various parameters and discuss their significance. Finally, in Sect. 7, we present concluding remarks.

\section{Basic hydrodynamic equations}

We consider a wedge shaped, axi-symmetric, low angular momentum flow (Chakrabarti 1990) with the number density distribution

$n(x)=\frac{\dot{M}}{\Omega m_{\mathrm{p}} x^{2} v(x)}$.

Here, $\Omega$ is the solid angle subtended by the flow

$\Omega=4 \pi \cos (\Theta)$,

where $\Theta$ is the angle made by the surface of the flow with the vertical axis, $m_{\mathrm{p}}$ the mass of the proton, $x$ the radial distance $r$ in units of $r_{\mathrm{g}}$ and $\dot{M}$ the accretion rate. The radial velocity

$v(x) \sim(x-1)^{-1 / 2}$

increases rapidly in this sub-Keplerian flow. In the presence of pure hydrogen, Thomson scattering will be the most dominating scattering process and the corresponding optical depth $\tau(x)$ can be computed using Eqs. (1) and (3). In the absence of any satisfactory description of the magnetic field in accretion disks, we assume only a random or stochastic field of strength $B(r)$, which may or may not be in equipartition with the ionized flow. We define a parameter $\beta$ as the ratio of the magnetic energy density and the gravitational energy density

$\beta=\frac{B^{2}\left(r-2 G M / c^{2}\right)}{8 \pi G M \rho}$.

Normally, $\beta \lesssim 1$ and for $\beta>1$, the magnetic field may buoyantly leave the disk.

The ions are expected to supply Coulomb energy to the electrons, which in turn become cooler using thermal and magnetic bremsstrahlung processes. In the presence of soft photons, they can loose energy via inverse Compton processes.

The energy balance equation for the protons and electrons in our model could be written as

$\frac{\mathrm{d} T_{\mathrm{p}}}{\mathrm{d} x}+\frac{T_{\mathrm{p}}(3 x-4)}{3 x(x-1)}+\frac{\Omega m_{\mathrm{p}}}{k \dot{M}} \frac{2}{3} x^{2}\left(\Gamma_{\mathrm{p}}-\Lambda_{\mathrm{p}}\right)=0$
$\frac{\mathrm{d} T_{\mathrm{e}}}{\mathrm{d} x}+\frac{3}{2}\left(\gamma_{\mathrm{pol}}-1\right) \frac{T_{\mathrm{e}}(3 x-4)}{3 x(x-1)}+\frac{\Omega m_{\mathrm{p}}}{k \dot{M}}\left(\gamma_{\mathrm{pol}}-1\right) x^{2}\left(\Gamma_{\mathrm{e}}-\Lambda_{\mathrm{e}}\right)=0$, 
where $\gamma_{\mathrm{pol}}$ is $5 / 3$ for non-relativistic electron temperatures $\left(T_{\mathrm{e}} \leq m_{\mathrm{e}} c^{2} / k\right)$ and $4 / 3$ for relativistic electron temperatures $\left(T_{\mathrm{e}}>m_{\mathrm{e}} c^{2} / k\right)$, and $k$ is the Boltzmann constant. Here the subscripts $\mathrm{p}$ and e stand for protons and electrons respectively. Since protons are much heavier than the electrons, $T_{\mathrm{p}}$ always remains in the non-relativistic domain. $\Gamma$ and $\Lambda$ contain contributions from all the heating and cooling processes respectively. We shall describe various terms shortly below.

One can integrate these equations simultaneously using a fourth order Runge-Kutta method. We impose as a condition that a steady accretion shock occurs at $x=x_{\mathrm{s}}$ such that the energy flux and the mass flux are conserved. In Chakrabarti (1989) it was shown that the shocks in a Schwarzschild geometry typically occur at around $x_{\mathrm{s}} \sim 10-80 r_{\mathrm{g}}$ depending on specific angular momentum $\lambda$. Instead of $\lambda$ we use $x_{\mathrm{s}}$ as a free parameter, along with $R$, the compression ratio. Normally, the shock location and the compression ratio are obtained simultaneously in a transonic solution (Chakrabarti 1989).

\section{Nature of the heating and cooling processes}

Protons lose energy through Coulomb interaction $\Lambda_{\text {ep }}$ and inverse bremsstrahlung $\Lambda_{\mathrm{ib}}$

$\Lambda_{\mathrm{p}}=\Lambda_{\mathrm{ep}}+\Lambda_{\mathrm{ib}}$

Electron-proton coupling supplies energy to the electrons (from protons) through $\Lambda_{\mathrm{ep}}$ which it lost due to various cooling processes. This behaves as a heating term as far as the electrons are concerned and is given by

$\Lambda_{\text {ep }}=1.6 \times 10^{-13} \frac{k \sqrt{m_{\mathrm{e}}} \ln \Lambda_{0}}{m_{\mathrm{p}}} n^{2}\left(T_{\mathrm{p}}-T_{\mathrm{e}}\right) T_{\mathrm{e}}^{-3 / 2}$,

where $\ln \Lambda_{0}$ is the Coulomb logarithm and $m_{\mathrm{p}}$ and $m_{\mathrm{e}}$ are the rest masses of protons and electrons respectively. Electrons are heated through this Coulomb coupling

$\Gamma_{\mathrm{e}}=\Lambda_{\mathrm{ep}}$.

Electrons are cooled by bremsstrahlung at the rate of $\Lambda_{b}$, by cyclo-synchrotron at the rate of $\Lambda_{\mathrm{cs}}$, and by Comptonization at the rate of $\Lambda_{\mathrm{mc}}$ of the soft photons due to cyclo-synchrotron radiation. The net cooling of the electrons is

$\Lambda_{\mathrm{e}}=\Lambda_{\mathrm{b}}+\Lambda_{\mathrm{cs}}+\Lambda_{\mathrm{mc}}$

Explicit expressions for these cooling terms for electrons satisfying Maxwell-Boltzmann (MB) distribution are

$$
\begin{aligned}
\Lambda_{\mathrm{ib}} & =1.4 \times 10^{-27} n^{2}\left(\frac{m_{\mathrm{e}}}{m_{\mathrm{p}}} T_{\mathrm{p}}\right)^{1 / 2}, \\
\Lambda_{\mathrm{b}} & =1.4 \times 10^{-27} n^{2} T_{\mathrm{e}}^{1 / 2}\left(1+4.4 \times 10^{-10} T_{\mathrm{e}}\right), \\
\Lambda_{\mathrm{cs}} & =\frac{2 \pi}{3 c^{2}} k T_{\mathrm{e}}(x) \frac{v_{\mathrm{a}}^{3}}{x},
\end{aligned}
$$

where $v_{\mathrm{a}}$ is the critical frequency at which the self-absorbed synchrotron radiation spectrum peaks and it can be determined from the relation

$v_{\mathrm{a}}=\frac{3}{2} v_{0} \theta_{\mathrm{e}}^{2} x_{\mathrm{m}}$ where

$$
\begin{aligned}
v_{0} & =2.8 \times 10^{6} B, \\
\theta_{\mathrm{e}} & =\frac{k T_{\mathrm{e}}}{m_{\mathrm{e}} c^{2}} .
\end{aligned}
$$

Procedure to determine $x_{\mathrm{m}}$ will be discussed below.

So far, we have discussed the situation when the electrons obey the MB velocity distribution law. However, in our present situation, a section of the flow passes through the shock and a fraction $\zeta$ of the electrons will have a power-law distribution. For instance, the cooling term due to cyclo-synchrotron photons is given by (Longair 1981)

$\Lambda_{c s}=\mathcal{A} G B^{(p+1) / 2}\left(v_{\max }^{(3-p) / 2}-v_{\min }^{(3-p) / 2}\right)$

where

$$
\begin{aligned}
\mathcal{A} & =\frac{(3 \pi)^{1 / 2} K e^{3}}{m_{\mathrm{e}} c^{2}(1+p)(3-p)}\left(\frac{2 \pi m_{\mathrm{e}}^{3} c^{5}}{3 e}\right)^{(1-p) / 2}, \\
\mathcal{G} & =\frac{\Gamma(p / 4+19 / 12) \Gamma(p / 4-1 / 12) \Gamma(p / 4+5 / 4)}{\Gamma(p / 4+7 / 4)}
\end{aligned}
$$

and where $K$ is the normalization constant of power-law electron distribution

$\left.n(\mathcal{E})\right|_{x_{\mathrm{s}}}=K \mathcal{E}^{-p}$

which is obtained using the constraint that the electron number is conserved during shock acceleration. Here $v_{\min }$ is the minimum frequency considered for integration. When selfabsorption is included, this may be treated as $v_{\mathrm{a}}$ defined above. Comptonization is computed by using this cooling term augmented by the enhancement factor $\mathcal{F}$

$\Lambda_{\mathrm{mc}}=\Lambda_{\mathrm{cs}} \mathcal{F}$.

For $\mathrm{MB}$ distribution, $\mathcal{F}$ is given by

$\mathcal{F}=\eta_{1}\left\{1-\left(\frac{x_{\mathrm{a}}}{3 \theta_{\mathrm{e}}}\right)^{\eta_{2}}\right\}$

where

$\eta_{1}=\frac{P(A-1)}{(1-P A)}$

and

$P=1-\exp \left(-\tau_{\mathrm{es}}\right)$

is the probability that an escaping photon is scattered.

$A=1+4 \theta_{\mathrm{e}}+16 \theta_{\mathrm{e}}^{2}$

is the mean amplification factor in the energy of a scattered photon when the scattering electrons have a Maxwellian velocity distribution of temperature $\theta_{\mathrm{e}}, \eta_{2}=1-\frac{\ln P}{\ln A}$ and $x_{\mathrm{a}}=$ $h v_{\mathrm{a}} / m_{\mathrm{e}} c^{2}$. The seed photons generated by the electrons obeying power-law distribution will also be comptonized by the thermal electrons, so that the amplification factor (Dermer et al. 1991) can be written as

$\mathcal{F}=\eta_{1}\left[1-\left(\frac{s}{\phi_{\mathrm{s}}}\right) \frac{x_{\max }^{\phi_{\mathrm{s}}}-x_{\mathrm{a}}^{\phi_{\mathrm{s}}}}{3 \theta_{\mathrm{e}}(\phi-1)\left(x_{\max }^{s}-x_{\mathrm{a}}^{s}\right.}\right]$, 
where

$$
\begin{aligned}
x_{\max } & =\frac{h v_{\max }}{m_{\mathrm{e}} c^{2}}, \\
s & =\frac{5-p}{2}, \\
\phi_{s} & =\phi+\frac{3-p}{2},
\end{aligned}
$$

and

$\phi=\eta_{2}-1$

Similarly, the amplification factor (Rybicki \& Lightman 1979) for the seed photons Comptonized by the electrons obeying power-law distribution can be written as

$\mathcal{F}=\frac{4}{3} \sigma_{\mathrm{T}} R_{\mathrm{c}} K \frac{\left(\mathcal{E}_{\max }^{3-p}-\mathcal{E}_{\min }^{3-p}\right)}{(3-p)}$

where $R_{\mathrm{c}}$ is the size of the Comptonized region. $\mathcal{E}_{\max }$ and $\mathcal{E}_{\min }$ are maximum and minimum energy of the power-law electrons, respectively.

In the current situation both thermal and non-thermal electrons are present. In this case, $x_{\mathrm{m}}$ is calculated by equating the total loss due to thermal and non-thermal emission from the CENBOL with contributions from the corresponding source functions from the CENBOL surface at the self-absorption frequency $v=v_{\mathrm{a}}$, i.e.,

$\left[\epsilon(v)_{\mathrm{MB}}+\epsilon(v)_{\mathrm{PL}}\right] \cdot V=\pi\left[(1-\zeta) \cdot p(v)_{\mathrm{MB}}+\zeta \cdot p(v)_{\mathrm{PL}}\right] \cdot S$,

where

$\epsilon(v)_{\mathrm{MB}}=4.43 \times 10^{-30} \frac{4 \pi n v}{K_{2}\left(1 / \theta_{\mathrm{e}}\right)} I\left(\frac{x_{\mathrm{m}}}{\sin (\psi)}\right)$.

$x_{m}$ is calculated from Eq. (11)

$x_{\mathrm{m}}=\frac{2 v}{3 v_{0} \theta_{e}^{2}}$,

and $\psi$ is the angle between the velocity vector of the electron and the direction of the local magnetic field. Averaging over $\psi$ for an isotropic velocity distribution, $I\left(\frac{x_{\mathrm{m}}}{\sin (\psi)}\right)$ gets replaced by $I^{\prime}\left(x_{\mathrm{m}}\right)$ and is given by (Narayan \& Yi 1995)

$I^{\prime}\left(x_{\mathrm{m}}\right)=\frac{4.0505}{x_{\mathrm{m}}{ }^{1 / 6}}\left(1+\frac{0.40}{x_{\mathrm{m}}{ }^{1 / 4}}+\frac{0.5316}{x_{\mathrm{m}}{ }^{1 / 2}}\right) \exp \left(-1.8899 x_{\mathrm{m}}{ }^{1 / 3}\right)$.

This is the emissivity of the electrons obeying MB distribution. The emissivity of the electrons obeying power-law distribution is given by

$\epsilon(v)_{\mathrm{PL}}=\frac{3-p}{2} \mathcal{A} G B^{(p+1) / 2}(v)^{(1-p) / 2}$.

The source function for thermal emission in the low frequency regime is given by

$p(v)_{\mathrm{MB}}=2 m_{\mathrm{e}} v^{2} \theta_{\mathrm{e}}$.

The source function of power-law emission is given by

$p(v)_{P L}=\frac{\sqrt{\pi}}{c^{2}(1+p)}\left(\frac{2 \pi m_{\mathrm{e}}^{3} c^{5}}{3 e}\right)^{1 / 2} \mathcal{H} B^{-1 / 2} v^{5 / 2}$, where

$\mathcal{H}=\frac{\Gamma(p / 4+19 / 12) \Gamma(p / 4-1 / 12) \Gamma(3 / 4)}{\Gamma(p / 4+11 / 6) \Gamma(p / 4+1 / 6) \Gamma(5 / 4)}$.

$\zeta$ is the percentage of electrons acquiring a power-law energy distribution. In Eq. (22), $V$ is the volume element under consideration, and $S$ is the corresponding surface area.

We have chosen to ignore the effects of power-law electrons on the emission of bremsstrahlung radiation since this radiation is very weak.

\section{Computation of the spectral index}

After showing how injected photon intensity is enhanced due to the inverse Comptonization process, this Section discusses how the spectral slope is computed. In CT95, the electrons in the pre-shock and the post-shock regions were assumed to follow Maxwell-Boltzmann distribution. Therefore the spectral index was computed from Titarchuk \& Lyubarskij (1995).

Electrons from the pre-shock flow will be accelerated at the shock and a fraction of post-shock electrons obey the powerlaw distribution such that the CENBOL has both the MB and power-law electrons. The energy spectral index $(\alpha)$ of the outgoing radiations due to Comptonization depends on the distribution function of electrons. We follow the prescription suggested in Titarchuk \& Lyubarskij (1995) and Gieseler \& Kirk (1997) for calculating $\alpha$, which is discussed below.

It is well known that a spectrum can be characterized by the dimensionless electron temperature $\theta_{\mathrm{e}}=k T_{\mathrm{e}} / m_{\mathrm{e}} c^{2}$ and the optical depth $\tau$ of the medium (Sunyaev \& Titarchuk 1985). The condition that has to be satisfied is

$C_{0}\left(\theta_{\mathrm{e}}, \alpha\right) \lambda\left(\tau_{0}\right)=1$

Here, $\lambda\left(\tau_{0}\right)=\exp (-\xi)$, and

$\xi=\frac{\pi^{2}}{3\left(\tau_{0}+2 / 3\right)^{2}}\left(1-\exp \left(-.7 \tau_{0}\right)+\exp \left(-1.4 \tau_{0}\right) \ln \frac{4}{3 \tau_{0}}\right.$,

for spherical geometry and $\tau_{0}$ is the total optical depth of the CENBOL. The quantity $C_{0}$ itself is to be obtained from

$C_{0}\left(\theta_{\mathrm{e}}, \alpha\right)=3 \pi \int_{0}^{1} v^{2} \mathrm{~d} v \frac{f(v)}{\gamma^{2}} \hat{C}_{0}$,

where $v, f(v)$, and $\gamma$ are the velocity of electrons in units of the velocity of light, electron velocity distribution function and the Lorentz factor, respectively, and

$\hat{C}_{0}=\frac{3}{8 \gamma^{2}}\left[I_{0,0}+I_{2,2}-\frac{1}{3}\left(I_{2,0}+I_{0,2}\right)\right]$.

The tabulated integrals $I_{i, j}$ is given by

$I_{i, j}=\int_{-1}^{1}(1+v x)^{\alpha} x^{i} \mathrm{~d} x \int_{-1}^{1} \frac{y^{j} \mathrm{~d} y}{(1+v y)^{\alpha+3}}$.

In the case of MB distribution

$f(v)=\frac{\gamma^{5} \exp \left(-\gamma / \theta_{\mathrm{e}}\right)}{4 \pi \theta_{\mathrm{e}} K_{2}\left(1 / \theta_{\mathrm{e}}\right)}$, 
where $K_{2}\left(1 / \theta_{\mathrm{e}}\right)$ is the modified Bessel function of second kind of order two and for power-law distribution (Eq. (15))

$f(v)=\frac{1-p}{4 \pi\left(\gamma_{\max }^{1-p}-\gamma_{\min }^{1-p}\right)} v \gamma^{3-p}$.

Here, $\gamma_{\max }$ and $\gamma_{\min }$ are obtained from particle energy as discussed in the next section. Integrating over different $f(v)$ we get different $\alpha$ for the same set of flow parameters. Using these $\alpha$ we calculate the spectrum from CENBOL as described in CT95.

\section{Solution procedure}

The geometry of the flow is chosen to be conical and the flow surface makes an angle $\Theta$ with the $z$-axis. We use the fourthorder Runge-Kutta method to integrate the energy Eqs. (5a) and (b). These two first order differential equations required two initial conditions when both flow geometry (determined mainly by the momentum equations) and accretion rate (determined by the continuity equation) are provided. At the shock, $x=x_{\mathrm{s}}$, the velocity $v(x)$ is reduced by a factor $R$, the compression ratio, i.e., $v_{+}\left(x_{\mathrm{s}}\right)=v_{-}\left(x_{\mathrm{s}}\right) / R$. The number density as obtained from Eq. (1) also goes up by a factor $R$.

As for the initial conditions, we fix the outer boundary at a large distance (say, $10^{6} r_{\mathrm{g}}$ ) and supply matter (both electrons and protons) with the same temperature (say, $T_{\mathrm{p}}=T_{\mathrm{e}}=$ $\left.10^{6} \mathrm{~K}\right)$. During integration, we obtained temperature and radiation emitted by the flow through bremsstrahlung and synchrotron radiation. These low energy photons are then inverse Comptonized by the hot electrons in the flow.

A shock of compression ratio $R$ causes the formation of power-law electrons of slope (Bell 1978a,b)

$p=(R+2) /(R-1)$.

These power-law electrons produce a power-law synchrotron emission with index $q$ given by (Longair 1981)

$q=(1-p) / 2$.

The power-law electrons have energy minimum at

$\mathcal{E}_{\min }=m_{\mathrm{e}} c^{2} \gamma_{\min }$

and energy maximum at

$\mathcal{E}_{\max }=m_{\mathrm{e}} c^{2} \gamma_{\max }$.

The $\mathcal{E}_{\max }$ was obtained self-consistently by conserving the number of power-law electrons and by computing the number of scatterings that the electrons undergo inside the disk before they escape. This yields,

$\gamma_{\max }=\gamma_{\min }\left[1+\frac{4}{3} \frac{R-1}{R} \frac{1}{x_{\mathrm{s}}^{1 / 2}}\right]^{x_{\mathrm{s}}^{1 / 2}}$.

The minimum value of the Lorentz factor $\gamma_{\min }$ is obtained from the temperature of the injected electrons, which is obtained self-consistently through our integration procedure. While obtaining the net cooling, we have used this local temperature and the local optical depth. To compute the spectrum, we divided the pre-shock region into several annuli and the post-shock region has been kept as a single zone. The number of intercepted soft-photons that participated in the Comptonization process has been calculated using local optical depth.

To obtain a realistic idea of how much matter actually passes through the shock, we resort to the numerical simulations of Molteni et al. (1994). This fraction depends on the angular momentum; for lower angular momentum, the flow passes close to the equatorial plane and passes through the shock formed very close to the hole. Very little matter is lost as wind. For higher angular momentum, the CENBOL size increases and a profuse amount of wind/outflow is formed after being processed through the CENBOL. The fraction can also be time-dependent. In the absence of a fully time-dependent solution, we assume the percentage of electrons $\zeta$ acquiring a power-law energy distribution to be a free parameter. The goal is to obtain spectra as varied as possible in order to constrain the parameters of a system.

In the presence of the magnetic field, the pre-shock flow and the CENBOL would emit synchrotron radiation. While computing this we have incorporated the synchrotron selfabsorption by the emitting medium when it becomes optically thick. Eq. (11) gives the expression for self-absorption frequency $v_{\mathrm{a}}$.

Though there are several approaches in the literature (see, e.g., Poutanen \& Coppi 1998; Gierliński et al. 1999; Coppi 1999; Zdziarski et al. 2001), we followed the procedures presented in CT95 and Titarchuk \& Lyubarskij (1995) while computing the Comptonized spectrum due to scattering from MB electrons and the power-law electrons. However, unlike CT95 where a Keplerian disk was supplying soft photons, here the source is everywhere, i.e., distributed throughout. This has been taken into account. At the end, we add contributions from all the components to get the net photon emissions from the flow.

In the next section, we present the spectral characteristics of the wedge-shaped thin flow around a $10 M_{\odot}$ black hole (unless otherwise stated) where $\Theta=75^{\circ}$. We generally use the strong shock $R=4$ except in Fig. 4 (where $R$ itself is varied) and saturated magnetic field $\beta=1$ except in Fig. 7 (where $\beta$ is varied). We vary other solution parameters $x_{\mathrm{s}}, \zeta$, and $\dot{m}$ to obtain results for different cases. Here, $\dot{m}$ is the mass accretion rate in units of Eddington rate $\dot{M}_{\text {Edd }}$. We generally use a black hole of mass $10 M_{\odot}$; however, we also probe the results when the mass was chosen to be $10^{6} M_{\odot}$.

\section{Results and interpretations}

First, we show the temperature distribution of incoming matter in the presence of cooling. Figures $1 \mathrm{a}$ and $\mathrm{b}$ show the variation of the electron and proton temperatures $\left(T_{\mathrm{e}}\right.$ and $\left.T_{\mathrm{p}}\right)$ as functions of the radial distance $x$ (measured in units of the Schwarzschild radius $r_{\mathrm{g}}$ ). Both axes are in logarithmic scale. Solid curves show the proton temperatures.

In Fig. 1a, results for $\dot{m}=0.01, M=10 M_{\odot}$ (long-dashed curve of $T_{\mathrm{e}}$ ) and $M=10^{6} M_{\odot}$ (dotted curve of $T_{\mathrm{e}}$ ) are shown. The other parameters are $\zeta=0.05$ and $x_{\mathrm{s}}=35$. For higher mass, density is lower and electrons are less efficiently cooled. 

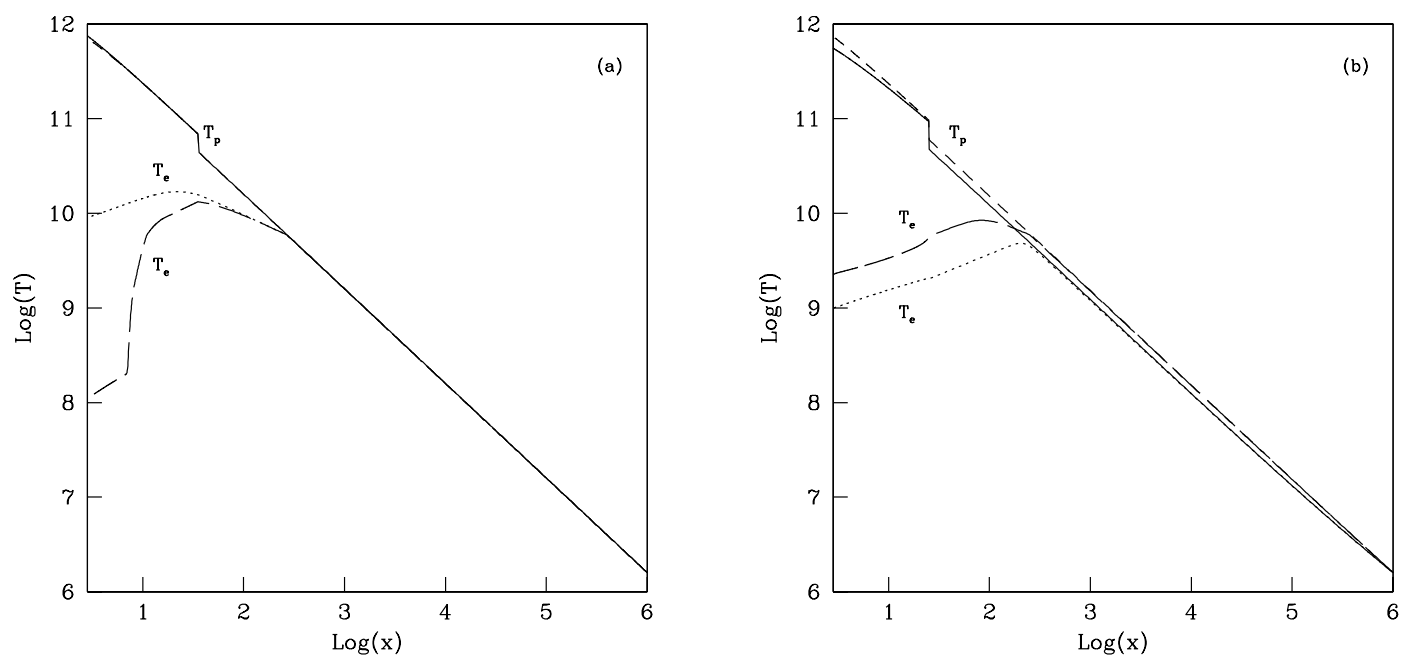

Fig. 1. Variation of the proton (solid) and electron temperatures as functions of dimensionless radial distance $x$ in presence of a strong shock $(R=4)$ with saturated magnetic field $(\beta=1)$. In a), $\dot{m}=0.01$ and $M=10 M_{\odot}$ (long-dashed) and $M=10^{6} M_{\odot}$ (dotted). Other parameters are $x_{s}=35$ and $\zeta=0.05$. For higher mass, the density becomes lower and the electrons are less efficiently cooled. In $\left.\mathbf{b}\right) \dot{m}=0.1$ (long-dashed) and $\dot{m}=0.5$ (dotted). Other parameters are $M=10 M_{\odot}, \zeta=0.2$ and $x_{\mathrm{s}}=25$. For higher accretion rates, the pre-shock flow cools faster and the temperatures deviate from each other farther away.
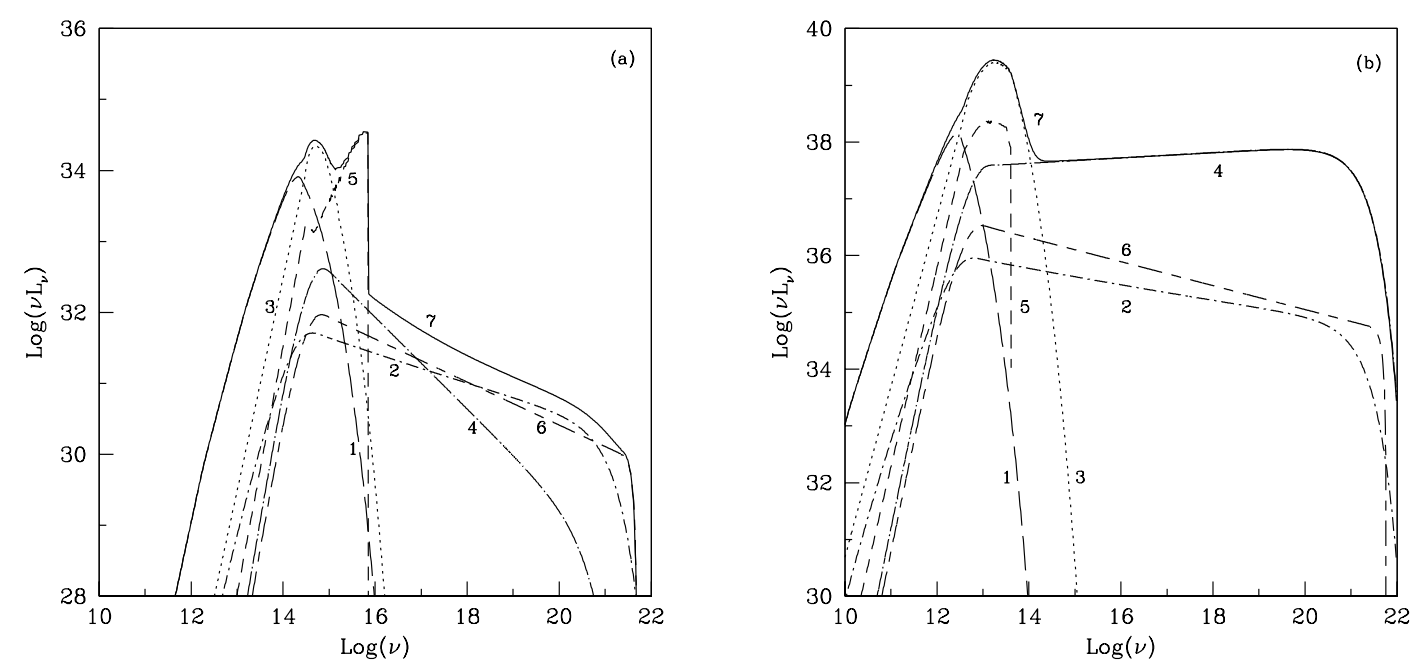

Fig. 2. Typical complete spectra from a sub-Keplerian accretion flow with all contributions shown for the same parameters as in Fig. 1a except for the black hole mass: a) $M=10 M_{\odot}$ and b) $M=10^{6} M_{\odot}$. See text for details of the components marked individually. In a), the spectrum is softer and in $\mathbf{b}$ ), the spectrum is harder due to lower density.

In Fig. $1 \mathrm{~b}, \dot{m}=0.1$ (long-dashed curve of $T_{\mathrm{e}}$ ) and $\dot{m}=0.5$ (dotted curve). Other parameters are $M=10 M_{\odot}, \zeta=0.2$, and $x_{\mathrm{s}}=25$. Higher accretion rate causes higher cooling of the electrons so that $T_{\mathrm{p}}$ and $T_{\mathrm{e}}$ start to deviate from each other farther from the black hole. Second, the initial deviation from proton temperature is also higher for higher rates. It is generally observed that just after the shock, electrons of the flow with lower accretion rate cool faster, because the CENBOL has more synchrotron photons to cool fewer electrons. Thus the role of the CENBOL is crucial to the quantitative change in the nature of cooling.

We now present a typical spectrum of an accretion flow around an $M=10 M_{\odot}$ black hole (Fig. 2a) and around an $M=10^{6} M_{\odot}$ black hole (Fig. 2b). Other parameters are exactly the same as in Fig. 1a. The curve marked 1 is due to the synchrotron emission by thermal electrons from the pre-shock region and that marked 2 is due to the Comptonization of these photons. These curves were drawn after self-absorption was taken into account. Curves 3 and 4 show synchrotron emission by thermal electrons and Comptonization of these photons by the same in the post-shock region. Curve 5 represents synchrotron emission by power-law electrons in the post-shock region, while 6 indicates Comptonization of the power-law generated soft photons and 7 is the total spectrum emitted from the entire flow. Bumps in the spectrum at around $\log (v) \sim$ 14-16 are the distinct signatures of synchrotron emission from the CENBOL. The bump at lower energy is due to the thermal electrons and the bump at higher energy is due to the power-law electrons. The photons around $\log (v) \sim 18-22$ are produced mainly by Comptonization of the thermal and nonthermal electrons. When it is dominated by the emission from the non-thermal electrons, it also carries the signature of the 


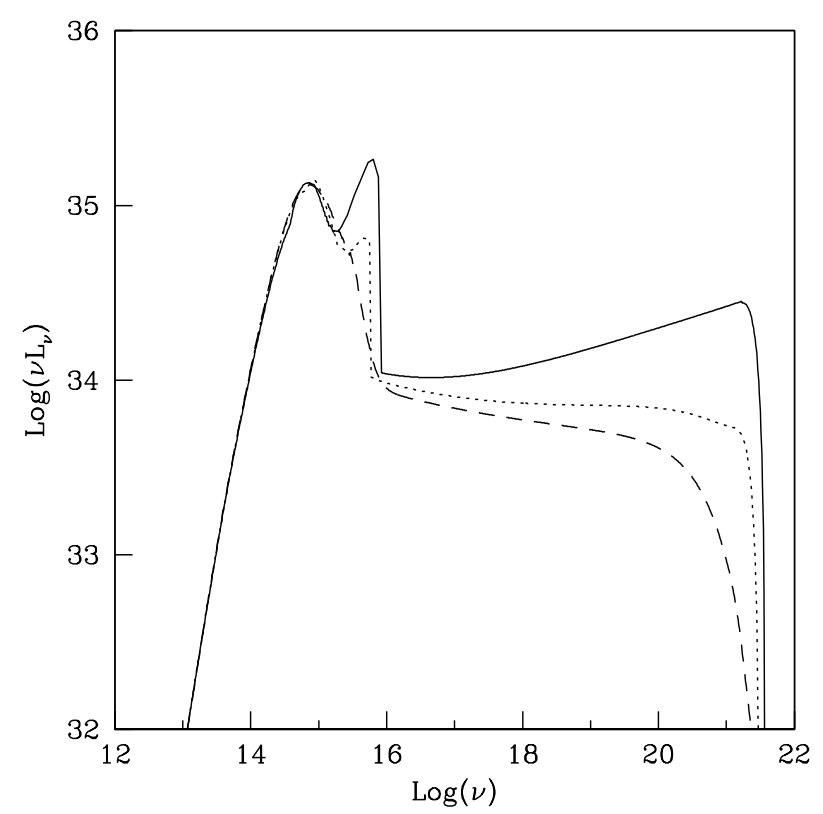

Fig. 3. Variation of the total emitted spectrum when shock location $x_{\mathrm{s}}$ is varied. The solid, dotted and dashed curves are drawn for $x_{\mathrm{s}}=$ 50,25 and 10 respectively. The spectrum becomes softer when $x_{\mathrm{s}}$ is reduced.

CENBOL. Notice in Curve 7, how spectral slope $\alpha$ changes from that of the thermal photons $\alpha_{\text {th }}$ to that of the non-thermal photons $\alpha_{\text {nth }}$. The major difference between the two cases is that in Fig. 2a, the spectrum is softer since the electron number density is higher for the low mass black hole, while in Fig. 2b the spectrum is harder because the electron number density is lower for the high mass black hole and because the electrons are difficult to cool by the synchrotron photons emitted by lower density electrons.

The location of the shock can change depending on the specific angular momentum and specific energy of the flow. When the shock is located at a larger distance, a much higher volume of the gas becomes hotter and the spectrum is expected to become harder. When the shock moves in, it is easy to cool the small CENBOL and the spectrum could become softer. We see this effect in Fig. 3 where we vary the shock location. The solid, dotted and the dashed curves denote the cases when $x_{\mathrm{s}}=50,25$ and 10, respectively. Other parameters are $\dot{m}=0.05$ and $\zeta=0.2$. When the shock location is very close the number of excursions by the electrons before escaping is lower so that $\gamma_{\max }$ is also lower (see, Eq. (37)). This causes the higher energy bump to disappear. When the shock is farther away, CENBOL radiation dominates the spectrum. These three cases can be thought of as representing an extremely rotating Kerr black hole with contra-rotating disk, a Schwarzschild black hole, and an extremely rotating Kerr black hole with corotating disk, in that order.

We show now that the extent to which pre-shock and post-shock flows dominate the spectral feature depends on the compression ratio of the shock. It is well known

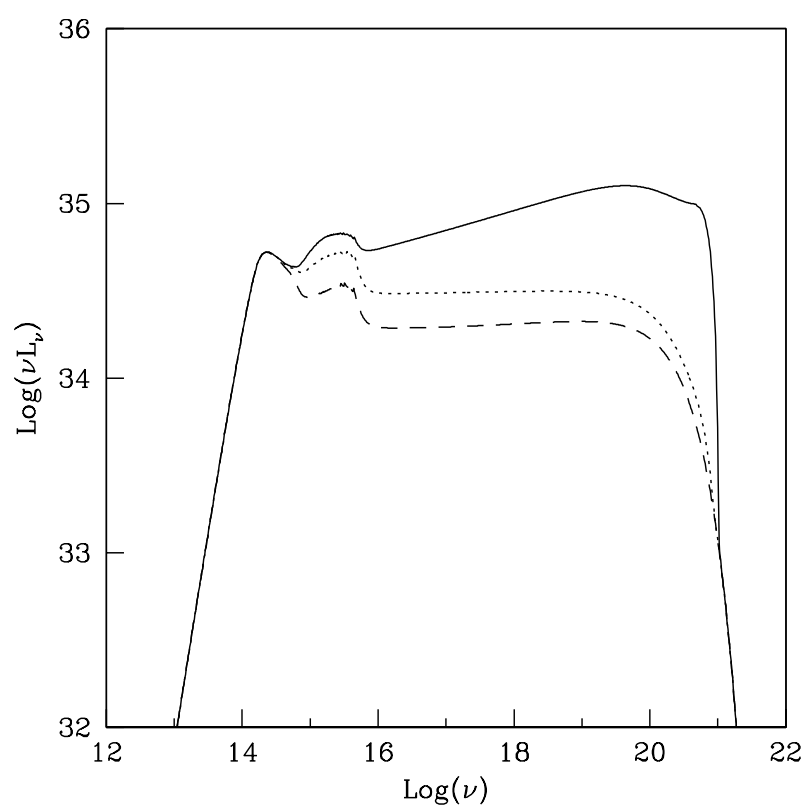

Fig. 4. Variation of the spectrum when compression ratio $R$ is varied. Solid, dotted and dashed curves are for $R=4.0,2.6$ and 1.5 respectively. In general, the spectrum hardens with increasing compression ratio.

(Landau \& Lifshitz 1956) that when the shock is strong, its compression ratio $R$ is given by

$R_{\text {strong }}=\frac{\gamma_{\mathrm{pol}}+1}{\gamma_{\mathrm{pol}}-1}$

where $\gamma_{\mathrm{pol}}$ is the polytropic index of the equation of state $P \propto \rho^{\gamma_{\mathrm{pol}}}$. In the case of mono-atomic gas, $\gamma_{\mathrm{pol}}=5 / 3$ and $R_{\text {strong }}=4$. However, for weak shocks, the strength may be $R \sim 2-3$. For the weakest shock (i.e., no shock), $R=1$. For an extremely relativistic flow, $\gamma_{\text {pol }}=4 / 3$ and the shock may have a strength up to 7 . In Fig. 4, we show the variation of the spectrum as a function of the compression ratio $R$. The accretion rate is $\dot{m}=0.5$ and $\zeta=0.2$. The solid curve is drawn for $R=4$; the dotted curve is for $R=2.6$ and the dashed curve is for $R=1.5$. For a weak shock, the jump in density $\rho_{+}=R \rho_{-}$, (where + and - denote the post and pre-shock values), at the shock is not so high and post-shock flow does not leave any distinct signature (long-dashed). The exactly opposite phenomenon occurs for a strong shock (solid). The high accretion rate means, it is difficult to cool the electrons, especially when the compression ratio is large, i.e., the electrons in the CENBOL are hotter. As a result, the spectrum is softer for $R=1.5$ and harder for $R=4$.

In Fig. 5, we show the spectrum's variation when $\dot{m}$ is varied. Here, $\zeta=0.2$ is used. A higher accretion rate is expected to increase the density of the flow, which enhances the magnetic field; therefore the synchrotron cooling is also increased. However, cooling is not sufficient when the optical depth enhancement is also taken into account. Thus, the spectrum becomes harder despite of this cooling. The Comptonized spectrum becomes harder. At lower accretion rate, it is easy to cool the flow and the spectrum becomes softer. The curves are drawn for: $\dot{m}=1.0$ (solid), 0.5 (dotted) and 0.1 (dashed). 


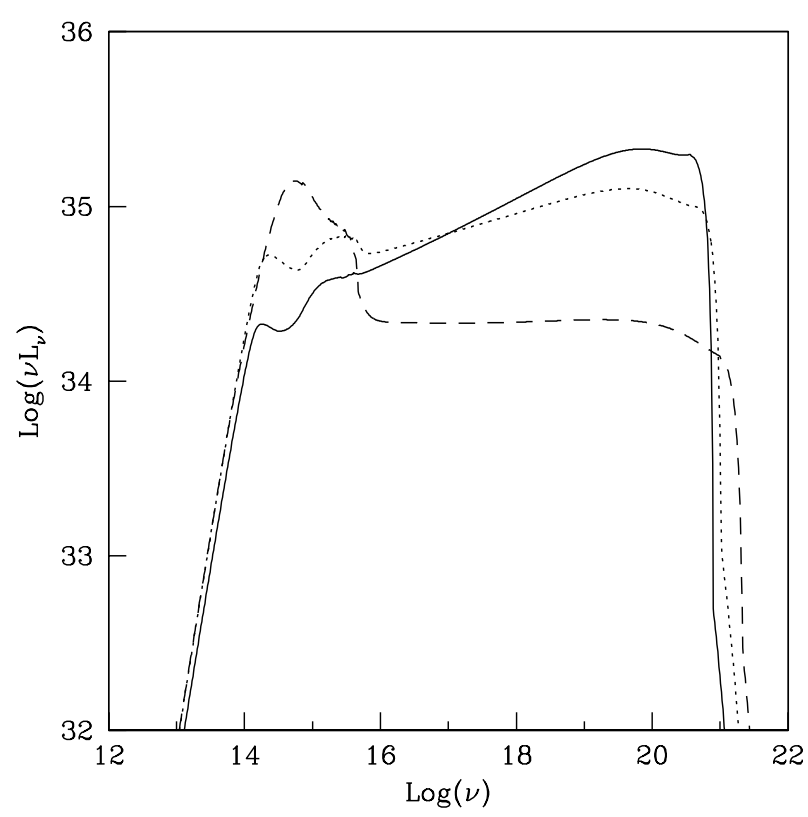

Fig. 5. Variation of the spectra with the dimensionless accretion rate. Solid, dotted and dashed curved are for $\dot{m}=1.0,0.5$ and 0.1 respectively. The spectrum becomes harder when the accretion rate is increased due to poor cooling effects.

As we discussed earlier, not all the accreting matter actually passes through the shock. Low angular momentum matter (for a given specific energy) produces a weaker jet, and if shock exists, most of the matter passes through the shock closer to the hole. High angular momentum inflow produces profuse outflows because the CENBOL size becomes very high. The fraction of electrons with power-law distribution $\zeta$ thus varies from 0 to 1 depending on the flow parameters. In Fig. 6, we show variation of the outgoing spectrum as a function of $\zeta$. The dashed curve is for $\zeta=0$, and the dotted and solid curves are for $\zeta=0.5$ and $\zeta=0.7$ respectively. We use $\dot{m}=0.05$ and $x_{\mathrm{s}}=25$. The spectrum changes from soft to hard with increase in $\zeta$. Note that the bump around $v \sim 10^{18} \mathrm{~Hz}$ is also weaker for smaller $\zeta$.

In all the cases mentioned above, we have assumed that the stochastic magnetic field is amplified sufficiently to become equipartition value at each radial distance. There are several reasons both for and against this argument. In the conical wedge shaped flow we used here, $B \sim 1 / r^{2}$ and the magnetic pressure $\sim 1 / r^{4}$ increases much faster compared with gas pressure $P_{\text {gas }} \sim 1 / r^{5 / 2}$. Thus, it is not unlikely that magnetic field, equipartition with gas pressure at a given outer radius, could remain in equipartition in all the radii by appropriately releasing magnetic energy. However, the time scale of amplification could be comparable to the buoyancy time-scale (Nandi et al. 2001) and it is not necessary that the low angular flow would get enough time to be amplified to the equipartition value. In the absence of any time-dependent simulation results in this direction, we put the ratio of magnetic energy to gravitational en$\operatorname{ergy}$, namely $\beta$, as a parameter, which enables us to understand the importance of the magnetic field. Since we are not considering any Keplerian disk, the only source of soft photon is synchrotron radiation, the intensity of which directly depends on

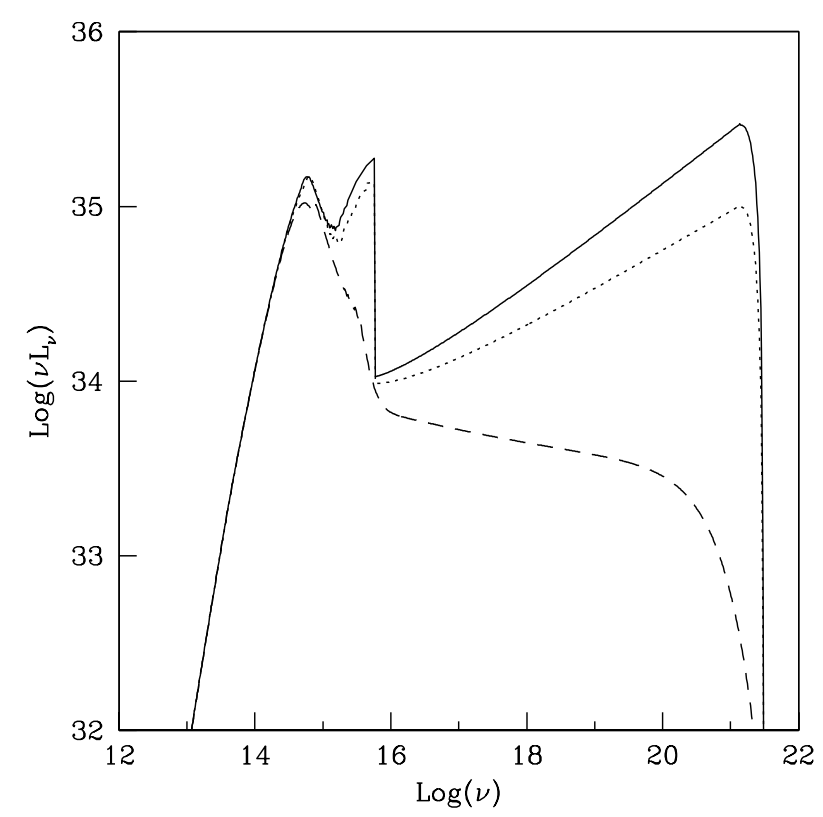

Fig. 6. Variation of the spectrum as a function of the percentage of electrons $(\zeta)$ which passes through an accretion shock. The dashed, dotted and solid curves are drawn for $\zeta=0.0,0.5$ and 0.7 respectively. The spectrum hardens with the increase in $\zeta$.

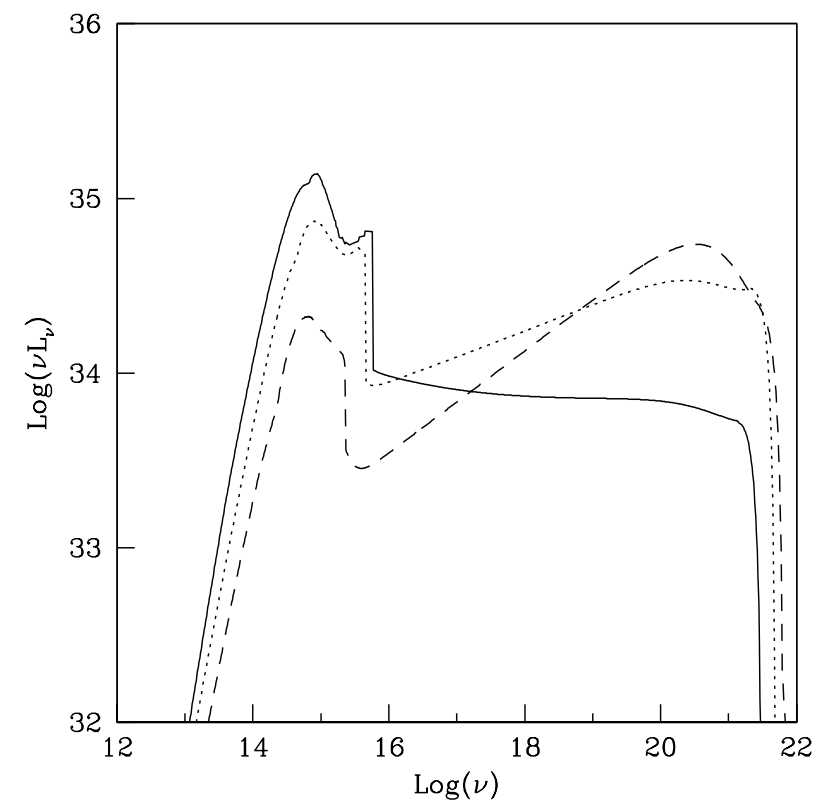

Fig. 7. Variation of the emitted spectrum when ratio $\beta$ between the magnetic energy density and the gravitational energy density is varied. Solid, dotted and dashed curves are for $\beta=1,0.1$ and 0.01 respectively. The spectrum softens when magnetic energy is increased.

the magnetic field. Figure 7 shows the spectral variation when $\beta$ is varied. The solid, dotted and the dashed curves are drawn for $\beta=1,0.1$, and 0.01 respectively. As expected, at low $\beta$, the spectrum is hard (dashed) and soft (solid) at high $\beta$. Thus, the spectral transition may also be triggered by the degree of magnetization in the flow.

While discussing Fig. 5, we have already mentioned that, when the accretion rate is increased, cooling takes place but the spectrum is hardened. This behaviour is generally seen in 

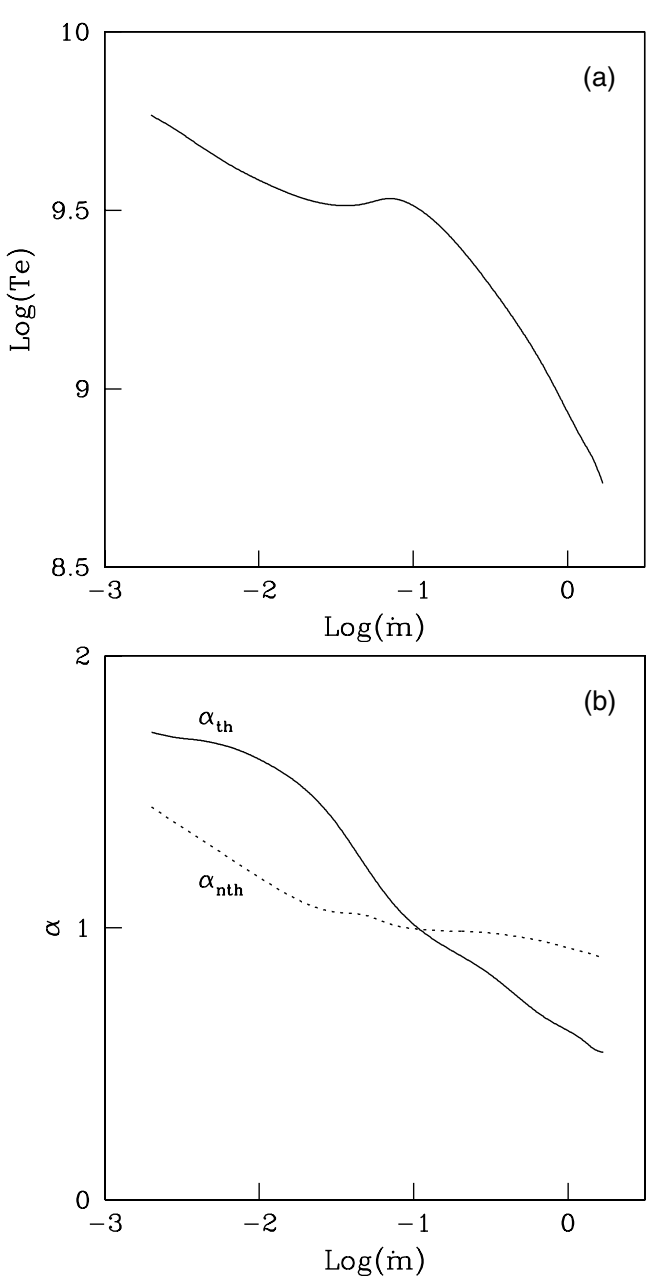

Fig. 8. Variation of the a) average electron temperature and b) thermal (solid) and non-thermal (dotted) spectral indices as functions of the mass accretion rate.

Figs. $8 \mathrm{a}$ and $\mathrm{b}$ where we present the variation of (a) the average electron temperature and (b) the spectral indices of the thermal $\left(\alpha_{\mathrm{th}}\right.$, solid $)$ and $\left(\alpha_{\mathrm{nth}}\right.$, dotted $)$ with the mass accretion rate. The parameters are $x_{\mathrm{s}}=35$ and $\zeta=0.1$. In this case, a bump is seen around $\dot{m} \sim 0.1$ where the electron temperature rises momentarily. Below this bump, synchrotron cooling dominates while above it Compton cooling dominates. Close to the bump these are of comparable magnitude. Both the thermal and non-thermal electrons show similar behaviour, namely, hardening with increase in accretion rate. This is markedly different from the CT95 result where the flow became softer with the accretion rate. This is because here we are plotting the variation with the halo rate (sub-Keplerian flow), while in CT95 the Keplerian rate was considered. There, too, it was found that the spectral index goes down with increase in the halo rate. Thus the behaviour in presence of synchrotron radiation (not present in CT95) is qualitatively similar.

\section{Discussion and concluding remarks}

In this paper, we have highlighted one major issue: to consider the effect of accretion shocks on spectral properties. To our knowledge, no such studies exist in the literature. An accretion shock separates a flow into two parts: the pre-shock flow is of lower density but higher volume, while the post-shock flow (CENBOL) is of higher density but lower volume. More important, the shock accelerates particles and generates non-thermal electrons. We consider this generation self-consistently. It is true that we use shock location and compression ratio as independent parameters, but since we did not explicitly use the angular momentum of matter, this re-parametrization is totally consistent. In this way, we thus incorporate the emission from the Maxwell-Boltzmann electrons and the power-law electrons, as well as Comptonization of these emissions. Since power-law electrons are present in the CENBOL, the radiation from these electrons is also the signature of the CENBOL.

In this paper, we have shown that, generally speaking, the spectral transition from a hard-state to a soft-state can be triggered by (a) reducing shock location (Fig. 3), (b) by reducing compression ratio (Fig. 4), (c) by reducing the accretion rate (Fig. 5), (d) by reducing power-law electron contents (Fig. 6), and (e) by increasing magnetic field strength inside the flow (Fig. 7). Note that, so far, we did not introduce the soft-photons from a Keplerian disk as was done in CT95. There, we did see that for a given rate of the Keplerian flow, if the accretion rate of the halo (sub-Keplerian flow) is increased, the spectrum becomes harder - an effect reflected in Fig. 5 above even when synchrotron photons are present. In the future, we shall include Keplerian disks as well. We shall also compare our results with observed spectra in order to pinpoint signatures of shocks in an accretion flow. In the presence of a Keplerian disk, the soft $\mathrm{X}$-ray bump will be produced around a few $\mathrm{KeV}$, while for shocks the bump due to Maxwell-Boltzmann electrons may be located in UV and that due the power-law electrons may accentuate already existing thermal bump located at a few hundred $\mathrm{keV}$ to a few MeV. Thus, it is possible that these two distinct bumps can be distinguished through observation.

The outflows produced from a CENBOL itself can have shocks. The shocks in outflows are located very close to the black holes, so that a pre-shock flow has very little volume. Post-shock outflows cool off rapidly and the cooler electrons $\left(T<10^{6} \mathrm{~K}\right)$ can also produce a low-energy, power-law spectrum. On the other hand, there could be several shocks in the jets. Their effects from our CENBOL will be discussed elsewhere.

Acknowledgements. The authors would like to thank the anonymous referee for suggestions leading to improvements in the paper. This work is partly supported by a grant from the RESPOND project of the Indian Space Research Organization.

\section{References}

Bell, A. R. 1978a, MNRAS, 182, 147

Bell, A. R. 1978b, MNRAS, 182, 443

Bondi, H. 1952, MNRAS, 112, 195

Chakrabarti, S. K. 1989, ApJ, 347, 365 
Chakrabarti, S. K. 1990, Theory of Transonic Astrophysical Flows (Singapore: World Scientific)

Chakrabarti, S. K. 1996, Phys. Rep. 266, 229

Chakrabarti, S. K., \& Manickam, S. G. 2000, ApJ, 531, L41

Chakrabarti, S. K., \& Molteni, D. 1995, 272, 80

Chakrabarti, S. K., \& Titarchuk, L. G. 1995, ApJ, 455, 623 (CT95)

Chakrabarti, S. K., Nandi, A., Manickam, S. G., Mandal, S., \& Rao, A. R. 2002, ApJ, 579, 21

Chakrabarti, S. K., Acharyya, K., \& Molteni, D. 2004, A\&A, 421, 1

Coppi, P. S. 1999, in High Energy Processes in Accreting Black Holes, ed. J. Poutanen, \& R. Svensson (San Francisco: ASP), ASP Conf. Ser., 161,375

Dermer, C. D., Liang, E. P., \& Canfield, E. 1991, ApJ, 369, 410

Eardley, D., \& Lightman, A. P. 1975, ApJ, 200, 187

Eardley, D., Lightman, A. P., \& Shapiro, S. L. 1975, ApJ, 199, 153

Eggum, G. E., Coroniti, F. V., \& Katz, J. I. 1985, ApJ, 298, L41

Esin, A. A., Narayan, R., Cui, W., Grove, J. E., \& Zhang, S.-N. 1998, ApJ, 505, 854

Galeev, A. A., Rosner, R., \& Vaiana, G. S. 1979, ApJ, 229, 318

Gierliński, M., Zdziarski, A. A., Poutanen, J., et al. 1999, MNRAS, 309,496

Gieseler, U. D. J., \& Kirk, J. G. 1997, A\&A, 323, 259

Kazanas, D., \& Elision, J. 1986, ApJ, 384, 1986

Landau, L., \& Lifshitz, E. M. 1959, Fluid Mechanics (New York: Pergamon Press)

Lin, D., \& Liang, E. P. 1999, A\&A, 341, 954

Longair, M. S. 1981, High Energy Astrophysics (UK: Cambridge Univ. Press)

Madau, P. 1988, ApJ, 327, 116
Mandal, S., \& Chakrabarti, S. K. 2004, Astrophys. \& Space Sc., Proceedings of the The Multiwavelength Approach To Unidentified Gamma-Ray Sources, ed. K. S. Chang, \& R. Romero (in press)

Molteni, D., \& Sponholz, H. 1994, MNRAS, 271, 233

Molteni, D., Lanzafame, G., \& Chakrabarti, S. K. 1994, ApJ, 425, 161

Molteni, D., Sponholz, H., \& Chakrabarti, S. K. 1996, ApJ, 457, 805

Molteni, D., Tóth, G., \& Kuznetsov, O. A. 1999, ApJ, 516, 411

Muchotrzeb, B., \& Paczynski, B. 1982, Acta Astron., 32, 1

Nandi, A., Chakrabarti, S. K., Vadawale S. V., \& Rao, A. R. 2001, A\&A, 380, 245

Narayan, R., \& Yi, I. 1995, ApJ, 452, 710

Paczyński, B., \& Wiita, P. J. 1980, A\&A, 88, 23

Poutanen, J., \& Coppi, P. S. 1998, Physica Scripta, T77, 57

Rees, M. J., Begelman, M. C., Blandford, R. D., \& Phinney, E. S. 1982, Nature, 295, 17

Ryu, D., Chakrabarti, S. K., \& Molteni, D. 1997, ApJ, 474, 378

Shakura, N. I., \& Sunyaev, R. 1973, A\&A, 24, 337

Shapiro, S. L. 1973a, ApJ, 180, 531

Shapiro, S. L. 1973b, ApJ, 185, 69

Shapiro, S. L., Lightman, A. P., \& Eardley, D. 1976 ApJ, 204, 187

Smith, D. M., Heindl, W. A., Markwardt, C. B., \& Swank, J. H. 2001, ApJ, 554, L41

Smith, D. M., Heindl, W. A., \& Swank, J. H. 2002, ApJ, 569, 362

Sunyaev, R. A., \& Titarchuk, L. 1985, A\&A, 143, 374

Titarchuk, L. G., \& Lyubarskij, Y. 1995, ApJ, 450, 876

Zdziarski, A. A., Grove, J. E., Poutanen, J., Rao, A. R., \& Vadawale, S. V. 2001, ApJ, 554, L45

Wardzinski, G., \& Zdziarski, A. A. 2001, MNRAS, 325, 963 